\title{
Impact of Medicaid insurance on outcomes following endoscopic transsphenoidal pituitary surgery
}

\author{
Iyan Younus, BS, ${ }^{1}$ Mina Gerges, MD, ${ }^{2}$ Theodore H. Schwartz, MD, ${ }^{2-4}$ and Rohan Ramakrishna, MD² \\ ${ }^{1}$ Weill Cornell Medical College; and Departments of ${ }^{2}$ Neurosurgery, ${ }^{3}$ Otolaryngology, and ${ }^{4}$ Neuroscience, Weill Cornell Medical \\ College, NewYork-Presbyterian Hospital, New York, New York
}

\begin{abstract}
OBJECTIVE Despite the rise of studies in the neurosurgical literature suggesting that patients with Medicaid insurance have inferior outcomes, there remains a paucity of data on the impact of insurance on outcomes after endonasal endoscopic transsphenoidal surgery (EETS). Given the increasing importance of complications in quality-based healthcare metrics, the objective of this study was to assess whether Medicaid insurance type influences outcomes in EETS for pituitary adenoma.
\end{abstract}

METHODS The authors analyzed a prospectively acquired database of EETS for pituitary adenoma from 2005 to 2018 at NewYork-Presbyterian Hospital, Weill Cornell Medicine. All patients with Medicaid insurance were identified. As a control group, the clinical, socioeconomic, and radiographic data of all other patients in the series with non-Medicaid insurance were reviewed. Statistical significance was determined with an alpha $<0.05$ using Pearson chi-square and Fisher's exact tests for categorical variables and the independent-samples t-test for continuous variables.

RESULTS Of 584 patients undergoing EETS for pituitary adenoma, 57 (10\%) had Medicaid insurance. The maximum tumor diameter was significantly larger for Medicaid patients (26.1 \pm 12 vs $23.1 \pm 11 \mathrm{~mm}$ for controls, $p<0.05)$. Baseline comorbidities including diabetes mellitus, hypertension, smoking history, and BMI were not significantly different between Medicaid patients and controls. Patients with Medicaid insurance had a significantly higher rate of any complication ( $14 \%$ vs $7 \%$ for controls, $p<0.05)$ and long-term cranial neuropathy $(5 \%$ vs $1 \%$ for controls, $p<0.05)$. There were no statistically significant differences in endocrine outcome or vision outcome. The mean postoperative length of stay was significantly longer for Medicaid patients compared to the controls $(9.4 \pm 31$ vs $3.6 \pm 3$ days, $p<0.05)$. This difference remained significant even when accounting for outliers (5.6 \pm 2.5 vs $3.0 \pm 2.7$ days for controls, $p<0.05)$. The most common causes of extended length of stay greater than 1 standard deviation for Medicaid patients were management of perioperative complications and disposition challenges. The rate of 30 -day readmission was $7 \%$ for Medicaid patients and $4.4 \%$ for controls, which was not a statistically significant difference.

CONCLUSIONS The authors found that larger tumor diameter, longer postoperative length of stay, higher rate of complications, and long-term cranial neuropathy were significantly associated with Medicaid insurance. There were no statistically significant differences in baseline comorbidities, apoplexy, endocrine outcome, vision outcome, or 30-day readmission.

https://thejns.org/doi/abs/10.3171/2020.1.JNS192707

KEYWORDS pituitary adenoma; endonasal; endoscopic; transsphenoidal; insurance; socioeconomic; complications; pituitary surgery

$\mathrm{H}$ EALTHCARE disparities have been reported in the neurosurgical literature, with patients covered by Medicaid experiencing significantly worse outcomes after surgery. ${ }^{1,2}$ It has also been reported that patients covered by Medicaid have significantly longer postoperative length of stay and total costs associated with their care. ${ }^{3}$ Therefore, there has been increased interest in analyzing socioeconomic disparities within neurosurgery.
Curry et al. analyzed a large nationwide sample of patients undergoing craniotomy procedures and found that patients covered by Medicaid had significantly higher rates of postoperative in-hospital mortality. ${ }^{1}$ Furthermore, El-Sayed and colleagues reported that patients covered by Medicaid had a significantly increased risk for postoperative complications within 30 days of neurosurgery. ${ }^{4}$

It has been theorized that these differences may be due

ABBREVIATIONS ACTH = adrenocorticotropic hormone; EETS = endonasal endoscopic transsphenoidal surgery; GH = growth hormone; GTR = gross-total resection; PE = pulmonary embolism.

SUBMITTED October 3, 2019. ACCEPTED January 16, 2020

INCLUDE WHEN CITING Published online March 20, 2020; DOI: 10.3171/2020.1.JNS192707. 
to baseline health, access to primary care, and access to postoperative outpatient follow-up care and rehabilitation. ${ }^{5}$ Despite the rise of studies in the neurosurgical literature suggesting that patients with Medicaid insurance have inferior outcomes, there remains a paucity of data on the impact of Medicaid insurance on outcomes after endonasal endoscopic transsphenoidal surgery (EETS). Given the increasing importance of complications in quality-based healthcare metrics, the objective of this study is to assess whether Medicaid insurance type influences outcomes in EETS for pituitary adenoma.

\section{Methods}

\section{Study Design}

The authors analyzed a prospectively acquired database of EETS for pituitary adenoma from 2005 to 2018 at NewYork-Presbyterian Hospital, Weill Cornell Medicine. We identified all patients with Medicaid insurance. As a control group, we also reviewed clinical, socioeconomic, and radiographic data of all other patients in the series with non-Medicaid insurance. The study was approved by our IRB.

\section{Tracking of Pre- and Perioperative Variables}

Preoperative clinical and radiographic data were collected and analyzed in both groups. Demographic parameters recorded for each case included sex, age, and postoperative length of stay. Tumor characteristics recorded for each case included maximum tumor diameter, cavernous sinus invasion determined by Knosp grade, radiographic extent of resection based on review of postoperative MRI by a board-certified neuroradiologist performed within a week after surgery, reoperation, apoplexy, and breakdown by functional adenoma subtype. Endocrine outcomes were recorded for both nonfunctioning and functioning adenomas. Complete biochemical remission for prolactinomas was determined by normalization of serum prolactin levels $<20 \mu \mathrm{g} / \mathrm{L}$; for growth hormone $(\mathrm{GH})$-producing tumors by normalization of serum $\mathrm{GH}<1 \mu \mathrm{g} / \mathrm{L}$ or nadir $\mathrm{GH}$ after an oral glucose tolerance test $<0.4 \mu \mathrm{g} / \mathrm{L}$; and for adrenocorticotropic hormone (ACTH)-producing tumors by morning serum cortisol $<3 \mu \mathrm{g} / \mathrm{dl}, 3-5$ days after surgery. Clinical history recorded for each case included preoperative vision status, preoperative cranial neuropathy, smoking status, BMI, history of diabetes mellitus, and history of hypertension. Socioeconomic factors assessed for each case included race/ethnicity (white, black, Hispanic, Asian, or other) and health insurance (Medicare, Medicaid, private, or self-pay). Imaging analysis was performed on a GE Advantage Workstation (version 4.3) to determine preoperative maximum cross-sectional length in any imaging plane.

\section{Tracking of Complications}

Complications were tracked in the immediate postoperative period and at long-term follow-up. Complications were categorized as infection, pulmonary embolism (PE), cranial neuropathy, vascular injury, CSF leak, hematoma, and readmission within 30 days.

\section{Statistical Analysis}

The SPSS statistical software package (version 24, IBM Corp.) was utilized for all analyses. Univariate analysis was performed using Pearson chi-square and Fisher's exact tests for categorical variables and the independentsamples t-test for continuous variables. For all tests, statistical significance was determined with an alpha $<0.05$.

\section{Results}

\section{Patient Demographics and Clinical Characteristics}

Among 584 patients undergoing EETS for pituitary adenoma, 10\% had Medicaid insurance (Table 1). Females comprised $51 \%$ of Medicaid cases compared to $49 \%$ of controls. The mean age for Medicaid cases was $49 \pm 14$ years compared to $52 \pm 16$ years for controls. Reoperations accounted for $12 \%$ of Medicaid cases compared to $13 \%$ of controls. Mean follow-up days were similar for both Medicaid and control cases (702 \pm 840 vs $711 \pm 844$ days, respectively). Race/ethnicity was not significantly different between Medicaid cases and controls. Among Medicaid cases, $53 \%$ were white, $16 \%$ black, $14 \%$ Hispanic, $5 \%$ Asian, and $12 \%$ classified as other, compared with $54 \%$ white, $19 \%$ black, $8.5 \%$ Hispanic, $7 \%$ Asian, and $11 \%$ other for the controls.

\section{Tumor Characteristics}

The maximum tumor diameter was significantly larger for Medicaid patients compared to the controls $(26.1 \pm 12$ vs $23.1 \pm 11 \mathrm{~mm}, \mathrm{p}<0.05)$. All other tumor characteristics were not significantly different between Medicaid cases compared to controls. Cavernous sinus invasion was present in 26\% of cases in the Medicaid group compared to $22 \%$ of the controls. Gross-total resection (GTR) was performed in $70 \%$ of cases in the Medicaid group compared to $72 \%$ of the controls. Functioning pituitary adenomas accounted for $32 \%$ of cases in the Medicaid group (22\% prolactinomas, $61 \% \mathrm{GH}$-producing, and 17\% ACTH-producing) compared to $27 \%$ of the controls ( $40 \%$ prolactinomas, $34 \%$ GH-producing, and 26\% ACTH-producing). Pituitary apoplexy was present in $7 \%$ of cases in the Medicaid group and $6 \%$ in the control group.

\section{Baseline Comorbidities}

Baseline comorbidities were not significantly different between Medicaid cases compared to the controls. History of diabetes mellitus and hypertension were present in $19 \%$ and $42 \%$ of Medicaid patients compared to $13 \%$ and $39 \%$ of the controls. Active smoking history was reported in $9 \%$ of Medicaid cases versus $8 \%$ in controls. The mean BMI was $28.7 \pm 6 \mathrm{~kg} / \mathrm{m}^{2}$ for Medicaid patients compared to $29.3 \pm 6 \mathrm{~kg} / \mathrm{m}^{2}$ for the controls.

\section{Length of Stay}

The mean postoperative length of stay was significantly longer for patients with Medicaid insurance $(9.4 \pm 3$ days) compared to controls $(3.6 \pm 3$ days, $\mathrm{p}<0.05)$. Accounting for outliers, the mean postoperative length of stay remained significant $(5.6 \pm 2.5$ days for Medicaid vs $3.0 \pm$ 2.7 days for controls, $\mathrm{p}<0.05)$. The most common causes 
TABLE 1. Univariate analysis of characteristics associated with Medicaid insurance

\begin{tabular}{lccc}
\hline \multicolumn{1}{c}{ Variable } & All Non-Medicaid (\%) & Medicaid Patients (\%) & p Value \\
\hline Demographics & & & \\
\hline No. of cases & $527(90)$ & $57(10)$ & \\
\hline Females/males & $256 \mathrm{~F}(49), 271 \mathrm{M}(51)$ & $29 \mathrm{~F}(51), 28 \mathrm{M}(49)$ & $\mathrm{NS}$ \\
\hline Mean age \pm SD, yrs & $52.4 \pm 16$ & $48.9 \pm 14$ & $\mathrm{NS}$ \\
\hline Mean length of stay \pm SD, days & $3.55 \pm 3$ & $9.4 \pm 31$ & $<0.05$ \\
\hline Reoperation & $68(13)$ & $7(12)$ & $\mathrm{NS}$ \\
\hline Mean follow-up \pm SD, days & $711 \pm 844$ & $702 \pm 840$ & $\mathrm{NS}$ \\
\hline Tumor characteristics & & & \\
\hline Max tumor diameter \pm SD, mm & $23.1 \pm 11$ & $26.1 \pm 12$ & $<0.05$ \\
\hline Cavernous sinus invasion & $115(22)$ & $15(26)$ & $\mathrm{NS}$ \\
\hline GTR & $380(72)$ & $40(70)$ & $\mathrm{NS}$ \\
\hline Functioning & $140(27)$ & $18(32)$ & $\mathrm{NS}$ \\
\hline Apoplexy & $31(6)$ & $4(7)$ & $\mathrm{NS}$ \\
\hline Comorbidities & & $11(19)$ & $\mathrm{NS}$ \\
\hline Diabetes mellitus & $70(13)$ & $24(42)$ & $\mathrm{NS}$ \\
\hline Hypertension & $204(39)$ & $5(9)$ & $\mathrm{NS}$ \\
\hline Active smoker & $44(8)$ & $28.7 \pm 6$ &
\end{tabular}

NS = nonsignificant.

Boldface type indicates statistical significance.

of extended length of stay greater than 1 standard deviation for Medicaid patients were management of perioperative complications and disposition challenges.

\section{Endocrine Outcome}

Nonfunctioning adenomas accounted for $73 \%$ of all pituitary adenomas (Table 2). The remainder were prolactinomas (10\%), GH-producing (10\%), and ACTH-producing (7\%). For nonfunctioning adenomas, $67 \%$ of Medicaid patients had normal postoperative endocrine status at last follow-up compared to $75 \%$ of controls. Among Medicaid patients, $100 \%$ of prolactinomas were cured biochemically, $82 \%$ of GH-producing adenomas were cured biochemically, and $67 \%$ of ACTH-producing adenomas were cured biochemically compared to $80 \%, 72 \%$, and $73 \%$ of controls, respectively. None of these differences in endocrine outcome were statistically significant.

\section{Pre- and Postoperative Vision and Cranial Neuropathy}

Preoperative vision was normal in 56\% and a deficit was present in $44 \%$ of Medicaid patients, compared to $57 \%$ normal and $43 \%$ with a deficit for the controls (Table 3). Postoperatively, 4 Medicaid patients (7\%) had a visual deficit compared to 14 (3\%) non-Medicaid patients. Preoperative cranial neuropathy was present in 3 Medicaid patients (5\%) compared to 15 non-Medicaid patients (3\%). None of these differences were statistically significant. Postoperatively, 3 Medicaid patients (5\%) had a cranial neuropathy compared to 6 non-Medicaid patients $(1 \%, \mathrm{p}<$ 0.05). Among Medicaid patients with cranial neuropathy, 2 had cranial nerve III palsy and 1 had cranial nerve VI palsy.

\section{Complications}

Patients with Medicaid insurance had a significantly higher rate of any complication (Table 4). The overall complication rate for Medicaid cases was $14 \%$ compared to $7 \%$ for the controls $(\mathrm{p}<0.05)$. Postoperative hematoma

TABLE 2. Endocrine outcomes for all pituitary adenoma subtypes

\begin{tabular}{cccc}
\hline Tumor Subtype & $\begin{array}{c}\text { All } \\
\text { Non-Medicaid } \\
(\%)\end{array}$ & $\begin{array}{c}\text { Medicaid } \\
\text { Patients } \\
(\%)\end{array}$ & p Value \\
\hline Nonfunctioning, $n=73 \%$ & & NS \\
\hline Normal & $289(75)$ & $26(67)$ & \\
\hline Panhypopituitarism & $53(14)$ & $9(23)$ & \\
\hline Mixed & $8(2)$ & $1(3)$ & \\
\hline Hypothyroidism & $5(1)$ & $1(3)$ & \\
\hline Hypogonadism & $28(7)$ & $2(5)$ & \\
\hline Hypocortisolism & $4(1)$ & 0 & NS \\
\hline Prolactinoma, $n=10 \%$ & & & \\
\hline Cured biochemically & $45(80)$ & $4(100)$ & \\
\hline Not cured & $11(20)$ & 0 & \\
\hline GH-producing, $n=10 \%$ & & & \\
\hline Cured biochemically & $34(72)$ & $9(82)$ & \\
\hline Not cured & $13(28)$ & $2(18)$ & \\
\hline ACTH-producing, $n=7 \%$ & & & \\
\hline Cured biochemically & $27(73)$ & $2(67)$ & \\
\hline Not cured & $10(27)$ & $1(33)$ & \\
\hline
\end{tabular}


TABLE 3. Pre- and postoperative vision and neurological deficits

\begin{tabular}{lccc}
\hline \multicolumn{1}{c}{ Variable } & $\begin{array}{c}\text { All Non-Medicaid } \\
(\%)\end{array}$ & $\begin{array}{c}\text { Medicaid } \\
\text { Patients (\%) }\end{array}$ & p Value \\
\hline Preop vision & & & NS \\
\hline Normal & $300(57)$ & $32(56)$ & \\
\hline Deficit & $227(43)$ & $25(44)$ & \\
\hline Postop vision & & & NS \\
\hline$\quad$ Normal & $513(97)$ & $53(93)$ & \\
\hline Deficit & $14(3)$ & $4(7)$ & \\
\hline Preop cranial neuropathy & & & NS \\
\hline Normal & $512(97)$ & $54(95)$ & \\
\hline Deficit & $15(3)$ & $3(5)$ & \\
\hline Postop cranial neuropathy & & & $<0.05$ \\
\hline$\quad$ Normal & $519(99)$ & $54(95)$ & \\
\hline Deficit & $6(1)$ & $3(5)$ & \\
\hline Bolfacetype &
\end{tabular}

Boldface type indicates statistical significance.

requiring reoperation occurred in 3.5\% of Medicaid cases compared to $1.3 \%$ of the controls. The 30 -day readmission rate for Medicaid cases was $7 \%$ compared to $4.4 \%$ for the controls. Meningitis, PE, vascular injury, and CSF leak occurred in none of the Medicaid cases. These rates were less than $1 \%$ in the controls; the rate of meningitis was $0.6 \%$, PE occurred in $0.2 \%$, vascular injury in $0.6 \%$, and CSF leak in $0.8 \%$.

\section{Causes of 30-Day Readmissions}

There were 4 unplanned 30-day readmissions (7\%) for Medicaid patients compared to 23 (4.4\%) for the controls, which was not a statistically significant difference. The most common cause of readmission was hyponatremia, which occurred in $75 \%$ of Medicaid patients and 57\% of controls. Other causes for readmission were epistaxis, sellar hematoma, spinal headache, pulmonary embolism, CSF leak, and meningitis.

\section{Discussion}

The proportion of Medicaid-insured patients in our cohort was $10 \%$, consistent with the rate of $6.7 \%-13.7 \%$ previously reported in the neurosurgical literature. ${ }^{1,4,6}$ Medicaid patients had no significant differences in baseline comorbidities including diabetes mellitus, hypertension, smoking history, and BMI. There were also no differences in endocrine outcome or vision outcome. However, Medicaid patients presented with significantly larger tumors and had a significantly higher rate of postoperative complications and long-term cranial neuropathy. Patients with Medicaid insurance also had a significantly longer postoperative length of stay, even when accounting for outliers.

There are limited reports in the pituitary literature investigating the impact of insurance type on outcomes and complications. Jahangiri et al. previously reported that socioeconomic factors associated with pituitary apoplexy are lack of health insurance and lack of a primary care provider. ${ }^{7}$ However, we found that the rate of apoplexy was not significantly different between Medicaid patients and
TABLE 4. Complications associated with Medicaid insurance

\begin{tabular}{lccc}
\hline \multicolumn{1}{c}{ Variable } & $\begin{array}{c}\text { All Non-Medicaid } \\
(\%)\end{array}$ & $\begin{array}{c}\text { Medicaid } \\
\text { Patients (\%) }\end{array}$ & $\begin{array}{c}\mathrm{p} \\
\text { Value }\end{array}$ \\
\hline Any complication & $36(7)$ & $8(14)$ & $<0.05$ \\
\hline Meningitis & $3(0.6)$ & 0 & $\mathrm{NS}$ \\
\hline PE & $1(0.2)$ & 0 & $\mathrm{NS}$ \\
\hline Cranial neuropathy & $6(1.1)$ & $3(5.3)$ & $<0.05$ \\
\hline Vascular injury & $3(0.6)$ & 0 & $\mathrm{NS}$ \\
\hline CSF leak & $4(0.8)$ & 0 & $\mathrm{NS}$ \\
\hline Hematoma & $7(1.3)$ & $2(3.5)$ & $\mathrm{NS}$ \\
\hline 30-day readmission & $23(4.4)$ & $4(7)$ & $\mathrm{NS}$ \\
\hline
\end{tabular}

Boldface type indicates statistical significance.

controls. Krings et al. analyzed state databases to examine factors associated with complications in pituitary surgery. ${ }^{8}$ They found that patients with Medicaid insurance were significantly more likely to have a complication (OR 2.13, 95\% CI 1.59-2.86). Goljo et al. also analyzed a nationwide cohort of patients undergoing pituitary surgery and found that African American and Hispanic patients have worse postoperative outcomes compared to white patients, as well as disproportionate utilization of Medicaid and lowvolume pituitary surgery centers. ${ }^{9}$ A significant limitation of the latter studies is that the presence of comorbidities and extent of disease cannot specifically be evaluated in these nationwide databases.

Similar trends are reported in the handful of reports investigating the impact of insurance type on outcomes and complications in the cranial neurosurgery literature. El-Sayed et al. analyzed a consecutive series of neurosurgery patients at their institution and found that postoperative complications among Medicaid patients were significantly higher than among privately insured patients (OR 3.1, 95\% CI 1.5-6.1). ${ }^{4}$ They also reported that Medicaid patients had significantly longer intensive care unit and hospital stays than privately insured patients $(\mathrm{p}<0.05)$. Chandra et al. recently conducted a study of 227 patients with glioblastoma and found that patients with Medicaid had higher surgical costs, longer lengths of stay, poorer survival, and lower quality-adjusted life year scores. ${ }^{6}$ They also found that Medicaid patients lack primary care providers, have more comorbidities, and present later in the disease course with larger tumors. Rong et al. reported that glioblastoma patients with Medicaid insurance have significantly shorter survival compared to those with nonMedicaid insurance after adjusting for confounding variables. ${ }^{10}$ They also found that uninsured patients were more likely to harbor larger tumors.

Curry et al. analyzed a nationwide cohort of 99,665 cranial operations and found that Medicaid patients had higher mortality rates compared to patients with private insurance. ${ }^{1}$ They also reported that Medicaid patients received care at lower-volume hospitals, whereas patients with private insurance received care at higher-volume centers. Curry et al. also found that race significantly impacted presentation and outcomes. In their study, African American patients generally presented with higher disease 
severity, including more emergency or urgent admissions; more hemiparesis and hemiplegia for primary tumors, meningiomas, and metastases; and more hydrocephalus for acoustic neuromas. Mukherjee et al. also reported on the prevalence of racial/ethnic disparities in neurosurgery; ${ }^{11}$ they found that African American and Hispanic patients have suboptimal access to tertiary care centers for neurooncology care.

The impact of insurance type is likely multifactorial and not completely understood. It has been reported that patients covered by Medicaid insurance lack access to quality primary care. ${ }^{1,5}$ This subset of patients is expected to present at a later stage of disease due to poor access to screening tests or imaging, often delaying diagnosis. This trend has been observed in glioblastoma and other malignancies in which Medicaid patients harbor larger tumors. ${ }^{12-14}$ In our study, we also found that Medicaid patients presented with significantly larger tumors. These are often more advanced and challenging to treat, and as a result, may lead to more complications, worse outcomes, and longer lengths of stay. We have previously reported that tumor diameter $\geq 30 \mathrm{~mm}$ and suprasellar extension are reliable predictors of postoperative complications, such as sellar hematomas requiring reoperation. ${ }^{15}$

In our cohort, Medicaid patients did not have significantly worse baseline comorbidities. These patients had similar rates of diabetes mellitus, hypertension, smoking history, and BMI. However, it is possible that unmeasured differences in baseline comorbidities may account for the variations in complication rates. We also did not find a significantly higher rate of cavernous sinus invasion or apoplexy in Medicaid patients. As a result, the higher rate of complications in these patients may be more likely due to presentation at a more advanced stage of disease as represented by a larger tumor size. However, other measures of disease severity such as duration of symptoms are not available in our database and represent a potential limitation.

Patients with Medicaid insurance had a significantly longer length of stay. Factors increasing length of stay may include increased perioperative complications, disposition challenges, and the cycle of longer hospitalization resulting in more nosocomial infections and complications. Disposition challenges may represent a unique challenge for Medicaid patients and have previously been associated with longer hospital stays. Gerszten et al. demonstrated that insurance barriers delay outpatient placement and transfers, negatively impacting health outcomes among neurosurgical patients. ${ }^{16,17}$ We found that the most common causes of extended length of stay greater than 1 standard deviation for Medicaid patients were management of perioperative complications and disposition challenges.

Patients who are uninsured or have Medicaid insurance are reportedly more likely to be younger, male, nonwhite, and unmarried.$^{10}$ In our cohort, we found no difference in age, sex, or race/ethnicity between the Medicaid patients and the controls. There is evidence from countries with universal coverage that socioeconomic gradients are independent predictors of healthcare outcomes. ${ }^{10}$ Therefore, poorer outcomes may be observed in patients with Medicaid insurance because it serves as a proxy for lower so- cioeconomic status. Based on this premise, it is possible that patients with Medicaid insurance may not have the financial capacity and social support to access high-quality home and hospital care, which may result in poorer outcomes. Furthermore, patients with Medicaid may lack coverage to support postoperative care and follow-up after discharge. However, our database was not initially designed to investigate social determinants of disease, and we do not have data regarding ZIP code and Medicaid proportion rates. We also do not have access to data regarding ease of access to hospital facilities or available social support for transport, etc. Certainly, these sorts of measures would be valuable but are not available and represent a potential limitation.

The findings of this study have important implications for informing healthcare policy and neurosurgical practice. We found that the effects of health insurance not only include access to care but extend beyond the initial hospitalization. Despite patients receiving the same neurosurgical care in our study, these patients had significantly worse outcomes. Although these patients did not have any significant differences in baseline comorbidities, they presented at a significantly more advanced stage of disease as evidenced by larger tumor size. Improved access to not only specialists, but also primary care may improve early detection in these patients. Furthermore, our findings build on existing literature that has demonstrated barriers in access to postoperative outpatient services for these patients. From a policy perspective, efforts to improve length of stay data by streamlining processes related to rehabilitation placement are vital. Avoiding delays in admitting patients to rehabilitation has many benefits including shorter length of time needed in acute rehabilitation, improved functional recovery, and decreased risk of nosocomial infections..$^{18}$

\section{Limitations and Future Directions}

The limitations of this study are its retrospective design involving a single institution, which is subject to imprecision of the medical record system and inherent bias. However, this is a consecutive series of cases and the database was created prospectively so no cases are missing. Some patients may present to another facility for postoperative complications but we believe the impact of this is limited due to postoperative outpatient visits, where any complications or hospitalizations would be identified.

Type of insurance is partially age dependent because Medicare patients are, by definition, 65 years or older. Therefore, associations between insurance type and health may be confounded by age, although this difference was not statistically significant between groups in our study. Additionally, our cohort was exclusively composed of patients presenting to a high-volume academic tertiary care center. It has been reported that underinsured patients may be more likely to seek care at low-volume centers, where outcomes have been shown to be worse. ${ }^{19,20}$ As a result, it may be possible that our findings actually underestimate the true disparities in neurosurgical outcomes nationally. Furthermore, although the rate of Medicaid patients in our cohort is consistent with that reported in the literature, we recognize that the proportion of Medicaid patients varies 
greatly and may be greater at other tertiary academic centers.

Future multicenter prospective studies are required to assess the relationship between socioeconomic metrics such as insurance type and outcomes after neurosurgery. This will help further clarify the etiologies of differences in postoperative outcomes by type of insurance. Future studies should also consider other metrics of socioeconomic status such as education, income, and geography, which were not captured by our database.

\section{Conclusions}

In a study of 584 consecutive cases of EETS for pituitary adenoma, we found that $10 \%$ of patients were covered by Medicaid insurance. These patients had a significantly larger tumor diameter, longer postoperative length of stay, higher rate of complications, and long-term cranial neuropathy. There were no statistically significant differences in baseline comorbidities, apoplexy endocrine outcomes, vision outcomes, or 30-day readmissions.

\section{References}

1. Curry WT Jr, Carter BS, Barker FG II. Racial, ethnic, and socioeconomic disparities in patient outcomes after craniotomy for tumor in adult patients in the United States, 1988-2004. Neurosurgery. 2010;66(3):427-438.

2. Hoh BL, Rabinov JD, Pryor JC, et al. In-hospital morbidity and mortality after endovascular treatment of unruptured intracranial aneurysms in the United States, 1996-2000: effect of hospital and physician volume. AJNR Am J Neuroradiol. 2003;24(7):1409-1420.

3. LaPar DJ, Bhamidipati CM, Mery CM, et al. Primary payer status affects mortality for major surgical operations. Ann Surg. 2010;252(3):544-551.

4. El-Sayed AM, Ziewacz JE, Davis MC, et al. Insurance status and inequalities in outcomes after neurosurgery. World Neurosurg. 2011;76(5):459-466.

5. Berk ML, Schur CL. Access to care: how much difference does Medicaid make? Health Aff (Millwood). 1998;17(3):169_ 180.

6. Chandra A, Young JS, Dalle Ore C, et al. Insurance type impacts the economic burden and survival of patients with newly diagnosed glioblastoma [published online June 21, 2019]. J Neurosurg. doi:10.3171/2019.3.JNS182629

7. Jahangiri A, Clark AJ, Han SJ, et al. Socioeconomic factors associated with pituitary apoplexy. J Neurosurg. 2013;119(6):1432-1436.

8. Krings JG, Kallogjeri D, Wineland A, et al. Complications following primary and revision transsphenoidal surgeries for pituitary tumors. Laryngoscope. 2015;125(2):311-317.

9. Goljo E, Parasher AK, Iloreta AM, et al. Racial, ethnic, and socioeconomic disparities in pituitary surgery outcomes. Laryngoscope. 2016;126(4):808-814.

10. Rong X, Yang W, Garzon-Muvdi T, et al. Influence of insurance status on survival of adults with glioblastoma multiforme: a population-based study. Cancer. 2016;122(20):31573165 .
11. Mukherjee D, Zaidi HA, Kosztowski T, et al. Disparities in access to neuro-oncologic care in the United States. Arch Surg. 2010;145(3):247-253.

12. Aizer AA, Falit B, Mendu ML, et al. Cancer-specific outcomes among young adults without health insurance. J Clin Oncol. 2014;32(19):2025-2030.

13. Grant SR, Walker GV, Guadagnolo BA, et al. Variation in insurance status by patient demographics and tumor site among nonelderly adult patients with cancer. Cancer. 2015;121(12):2020-2028.

14. Rosenberg AR, Kroon L, Chen L, et al. Insurance status and risk of cancer mortality among adolescents and young adults. Cancer. 2015;121(8):1279-1286.

15. Younus I, Gerges MM, Godil SS, et al. Incidence and risk factors associated with reoperation for sellar hematoma following endoscopic transsphenoidal pituitary surgery [published online August 23, 2019]. J Neurosurg. doi:10.3171/2019.6.JNS191169

16. Gerszten PC, Witham TF, Clyde BL, Welch WC. Relationship between type of health insurance and time to inpatient rehabilitation placement for surgical subspecialty patients. Am J Med Qual. 2001;16(6):212-215.

17. Taylor L, Simpson K, Bushardt R, et al. Insurance barriers for childhood survivors of pediatric brain tumors: the case for neurocognitive evaluations. Pediatr Neurosurg. 2006;42(4):223-227.

18. Gogstad AC, Kjellman AM. Rehabilitation prognosis related to clinical and social factors in brain injured of different etiology. Soc Sci Med. 1976;10(6):283-288.

19. Cowan JA Jr, Dimick JB, Leveque JC, et al. The impact of provider volume on mortality after intracranial tumor resection. Neurosurgery. 2003;52(1):48-54.

20. Solomon RA, Mayer SA, Tarmey JJ. Relationship between the volume of craniotomies for cerebral aneurysm performed at New York state hospitals and in-hospital mortality. Stroke. 1996;27(1):13-17.

\section{Disclosures}

The authors report no conflict of interest concerning the materials or methods used in this study or the findings specified in this paper.

\section{Author Contributions}

Conception and design: all authors. Acquisition of data: all authors. Analysis and interpretation of data: all authors. Drafting the article: all authors. Critically revising the article: all authors. Reviewed submitted version of manuscript: all authors. Approved the final version of the manuscript on behalf of all authors: Ramakrishna. Statistical analysis: all authors. Study supervision: Ramakrishna, Schwartz.

\section{Correspondence}

Rohan Ramakrishna: Weill Cornell Medical College, NewYorkPresbyterian Hospital, New York, NY. ror9068@med.cornell.edu. 